\title{
A Novel Synthetic UV-Curable Fluorinated Siloxane Resin for Low Surface Energy Coating
}

\author{
Chunfang Zhu ${ }^{1,+}{ }^{\dagger}$ Haitao Yang ${ }^{1,+}$, Hongbo Liang ${ }^{1, *}$, Zhengyue Wang ${ }^{1}$, Jun Dong ${ }^{1}$, Lei Xiong ${ }^{1}$, \\ Jianping Zhou ${ }^{1}$, Junjun $\mathrm{Ke}^{1}, \mathrm{Xi} \mathrm{Xu}^{1}$ and Weixian $\mathrm{Xi}^{2,3, *}$ \\ 1 School of Material Science and Engineering, Nanchang Hangkong University, Nanchang 330063, China; \\ Zhuchunfang_123@163.com (C.Z.); yht@nchu.edu.cn (H.Y.); 18270890654@163.com (Z.W.); \\ dj303@126.com (J.D.); xionglei@nchu.edu.cn (L.X.); zf161162@163.com (J.Z.); 18779160082@163.com (J.K.); \\ xuxi19930904@163.com (X.X.) \\ 2 Department of Chemical and Biomolecular Engineering, University of California Los Angeles, Los Angeles, \\ CA 90095, USA \\ 3 Department of Orthopaedic Surgery, University of California Los Angeles, Los Angeles, CA 90095, USA \\ * Correspondence: liahongbo@163.com (H.L.); wxi@mednet.ucla.edu (W.X.); Tel.: +86-0791-83863025 (H.L.) \\ + These authors contributed equally to this work.
}

Received: 20 July 2018; Accepted: 28 August 2018; Published: 3 September 2018

check for updates

\begin{abstract}
Low surface energy materials have attracted much attention due to their properties and various applications. In this work, we synthesized and characterized a series of ultraviolet (UV)-curable fluorinated siloxane polymers with various fluorinated acrylates-hexafluorobutyl acrylate, dodecafluoroheptyl acrylate, and trifluorooctyl methacrylate-grafted onto a hydrogen-containing poly(dimethylsiloxane) backbone. The structures of the fluorinated siloxane polymers were measured and confirmed by proton nuclear magnetic resonance and Fourier transform infrared spectroscopy. Then the polymers were used as surface modifiers of UV-curable commercial polyurethane (DR-U356) at different concentrations $(1,2,3,4,5$, and $10 \mathrm{wt} \%)$. Among three formulations of these fluorinated siloxane polymers modified with DR-U356, hydrophobic states $\left(91^{\circ}, 92^{\circ}\right.$, and $\left.98^{\circ}\right)$ were obtained at low concentrations ( $1 \mathrm{wt} \%)$. The DR-U356 resin is only in the hydrophilic state at $59.41^{\circ}$. The fluorine and siloxane element contents were investigated by X-ray photoelectron spectroscopy and the results indicated that the fluorinated and siloxane elements were liable to migrate to the surface of resins. The results of the friction recovering assays showed that the recorded contact angles of the series of fluorinated siloxane resins were higher than the original values after the friction-annealing progressing.
\end{abstract}

Keywords: ultraviolet (UV) curable coatings; low surface energy materials; fluorinated siloxane resin

\section{Introduction}

Low surface energy materials (LSEMs) have been widely investigated due to their self-cleaning, drag-reducing, stain-resisting, and anti-fouling properties, which have enabled LSEMs to be applied as food packaging, and protective or anti-adhesion coatings [1,2]. In the low surface energy coating fabrication process, the two most significant factors are chemical structure and appropriate surface roughness $[3,4]$. For the former, researchers usually use $-\mathrm{CF}_{3}$ and $-\mathrm{CF}_{2}$ functional groups to create low surface energy of $\sim 15 \mathrm{mN} \cdot \mathrm{m}^{-1}$ and $\sim 25 \mathrm{mN} \cdot \mathrm{m}^{-1}$, respectively [3]. However, the extensive use of fluorocarbon resins is limited by poor compatibility, environmental contamination, bioaccumulation, and toxicity issues [3-7]. Therefore, to overcome this limitation, in this work, we proposed that the introduction of siloxane could increase the compatibility of the polymer.

In numerous organo-silicon compounds, polysiloxane and siloxane have been frequently investigated and reported due to their low-surface energy [8]. Polysiloxane not only exhibits thermal 
stability, low-surface energy, and chemical resistance, but also has a lower glass transition temperature, as well as non-toxic and anti-biological attachment characteristics [9]. Zhan et al. [10] proposed using polysiloxane mixed with polycaprolactone as the soft segment, isophorone diisocyanate (IPDI) as the hard segment, and a siloxane coupling agent as the chain extender to synthesize the silicone-modified polyurethane. They demonstrated that the increase in organo-silicone compound concentration led to the increase in the contact angle of the material surface. Li et al. [9] used $\alpha, \omega$-hydroxy-terminated polydimethylsiloxane (HTPDMS' hydroxyl value $\geq 8 \%$ ) and methyltrimethoxysilane with a dibutyl tin dilaurate (DBTL) catalyst to create a superhydrophobic surface.

The copolymer consists of fluorinated polydimethylsiloxane segments that might yield high performance materials. Yan et al. [11], using hydroxy-terminated polydimethylsiloxane, dicarboxyl terminated poly(2,2,3,4,4,4-hexafluorobutyl acrylate) (CTHFA), toluene diisocyanate, and 2-hydroxyethyl methacrylate (HEMA), produced a new ultraviolet (UV)-cured vinyl-terminal fluorinated siloxane graft copolymer (Vi-PFSi). The coating surface energy decreased from $45.52 \mathrm{mN} / \mathrm{m}^{2}$ to $15.40 \mathrm{mN} / \mathrm{m}^{2}$ when the concentration of the Vi-PFSi was loaded at $0.5 \mathrm{wt} \%$. In this case, the hydrogen-containing polydimethylsiloxane was used as a functional spacer.

Based on the characteristics of the organo-silicon compound, here we designed and synthesized three types of fluorinated acrylate on organo-silicon [12-14]. The copolymer was synthesized from hydropolydimethylsiloxane, fluorinated acrylate, mercaptoethanol, acrylic acid, and 3-isocyanatomethyl-3,5,5-trimethylcyclohexyl isocyanate (IPDI) [15,16], which was then grafted to UV-cured fluoro-silicone resin. The surface properties of the modified UV-curable polyurethane were examined by contact angle measurements with water. The surface element contents were evaluated by X-ray photoelectron spectroscopy (XPS). In addition, the acid and alkali resistance were also evaluated in $10 \mathrm{wt} \% \mathrm{NaOH}$ and $10 \mathrm{wt} \% \mathrm{H}_{2} \mathrm{SO}_{4}$ solution. Our results indicate that this modified resin's chemical resistance and physical properties were significantly improved with $1 \mathrm{wt} \%$ modification with synthesized fluorosilicone. The novelty of this work was that the friction recovering assays showed that the series of fluorinated siloxane resin's contact angles recovered to higher than the original values after the friction-annealing progressing, making it a huge application prospect.

\section{Materials and Experiment}

\subsection{Materials}

Acrylic acid (AA; purity $\geq 99.8 \%$ ) was purchased from Xilong Chemical Co., Ltd. (Shantou, China). Allyl glycidyl ether (AGE; purity $\geq 99.5 \%$ ) and mercaptoethanol $(\geq 99.5 \%)$ were purchased from Aladdin Co. Isophorone diisocyanate (IPDI; Bayer Reagents Co., Ltd., Shenzhen, China), hexafluorobutyl acrylate (HFOA; represented by G01 in this article), dodecafluoroheptyl acrylate (DFOA; referred to as G05 in this article), and trifluorooctyl methacrylate (TFOMA; referred to as G06B in this article; purity $\geq 99.5 \%$ ) were obtained from Harbin Sunshine Fluorine Silicon Chemical Co., Ltd., Harbin, China). Hydrogen-containing polydimethylsiloxane (H-PDMS; purity $\geq 99 \%$ ) was purchased from Shenzhen Chonghuaxin Technology Co., Ltd. (Harbin, China). DR-U356 was obtained from Changxin Material Industry Limited by Share Ltd. (Harbin, China).

\subsection{Synthesis of Monomers}

\subsubsection{Synthesis of Hydroxyl-Containing Polydimethylsiloxane (OH-PDMS)}

The hydroxyl-containing polydimethylsiloxane was prepared via a two-step reaction. Firstly, $0.15 \mathrm{~mol}(17.1 \mathrm{~g}$ ) allyl glycidyl ether (AGE), an equal mass of toluene, and $300 \mathrm{ppm}$ of Kastedt's catalyst (according to the total mass of reactants) were added to a three-neck, round-bottom flask, heated to 65 (protected by $\mathrm{N}_{2}$ ). Then, the $50 \mathrm{~g}$ H-PDMS were slowly dropped into the bottom while the temperature was increased to $70{ }^{\circ} \mathrm{C}(6-8 \mathrm{~h})$. Vacuum distillation was used to remove extra AEG and toluene to obtain the epoxy polydimethylsiloxane (EPDMS) (Step 1, Figure 1). Secondly, $15 \mathrm{~g}$ EPDMS and the 
same mass of toluene were added to the three-neck, round-bottom flask, the mixture was heated to $75^{\circ} \mathrm{C}$ and held for $30 \mathrm{~min}$. After that, $0.0021 \mathrm{~mol} \mathrm{AA}, 1 \mathrm{wt} \%$ (the total mass of the reaction system) P-hydroxyanisole (MEHQ) and $0.413 \mathrm{~g} \mathrm{~N}, \mathrm{~N}$-dimethyl benzyl ammonia (DBMA) were dropped into the bottom, and the mixture was heated to $95^{\circ} \mathrm{C}$. NaOH- and $\mathrm{NaCl}$-saturated solutions were used to wash extra AA and MEHQ to obtain the hydroxyl-containing polydimethylsiloxane (OH-PDMS) (Step 2, Figure 1).



Figure 1. Synthesis of novel ultraviolet (UV)-curable fluorinated siloxane resins.

\subsubsection{Modification of Fluorinated Intermediaries (OH-G01, OH-G05, and OH-G06B)}

Using the synthesis of OH-G01 as an example, $0.01 \mathrm{~mol}$ fluorinated monomer hexafluorobutyl acrylate (HFOA) and the same mass of dimethylformamide (DMF) were added into a three-neck, round-bottom flask, the temperature was increased to $55^{\circ} \mathrm{C}$, and $0.02 \mathrm{~mol}$ mercaptoethanol mixed with $0.5 \mathrm{wt} \%$ triethylamine solution were dripped into the bottom over $30 \mathrm{~min}$. DMF and trimethylamine were removed after the reaction finished. OH-G05 and OH-G06 were synthesized using the same method (Step 3, Figure 1). 


\subsubsection{Synthesis of Graft Fluorinated Siloxane Polymers (Si-G01, Si-G05, and Si-G06B)}

Next, 0.01 mol IPDI, 0.01 mol fluorine intermediaries, and the same mass of toluene with the catalyst of $5 \mathrm{wt} \%$ DBTDL were added to the three-neck, round-bottom flask with magnetic stirring kept for about $2.5 \mathrm{~h}$ (Step 4, Figure 1). When the reaction was accomplished, a mixture of $0.01 \mathrm{~mol}$ hydroxyl-containing polydimethylsiloxane (OH-PDMS), an equal mass of toluene, and $5 \mathrm{wt} \%$ DBTDL were dropped into the flask slowly to obtain the Si-G01, Si-G05, and Si-G06B polymers (Step 5, Figure 1).

\subsection{Preparation of Samples}

\subsubsection{Resin Formulation}

The UV-cured coatings were prepared as follows: the synthesized Si-G01, Si-G05, and Si-G06B, and the same acetone and initiator (Irgacure 1173) were added to the matrix resin (DR-U356) to study the influence of the properties of the contents of the different additives on the matrix resin (Table 1).

Table 1. The composition of graft fluorinated siloxane resins.

\begin{tabular}{ccccccc}
\hline & Si-G01 (g) & Si-G05 (g) & Si-G06B (g) & DR-U356 (g) & Acetone (g) & Initiator (g) \\
\hline SG01-01 & 0.1 & $\times$ & $\times$ & 9.9 & 2.0 & 0.4 \\
SG01-02 & 0.2 & $\times$ & $\times$ & 9.8 & 2.0 & 0.4 \\
SG01-03 & 0.3 & $\times$ & $\times$ & 9.7 & 2.0 & 0.4 \\
SG01-04 & 0.4 & $\times$ & $\times$ & 9.6 & 2.0 & 0.4 \\
SG01-05 & 0.5 & $\times$ & $\times$ & 9.5 & 2.0 & 0.4 \\
SG01-10 & 1.0 & $\times$ & $\times$ & 9.0 & 2.0 & 0.4 \\
SG05-01 & $\times$ & 0.1 & $\times$ & 9.9 & 2.0 & 0.4 \\
SG05-02 & $\times$ & 0.2 & $\times$ & 9.8 & 2.0 & 0.4 \\
SG05-03 & $\times$ & 0.3 & $\times$ & 9.7 & 2.0 & 0.4 \\
SG05-04 & $\times$ & 0.4 & $\times$ & 9.6 & 2.0 & 0.4 \\
SG05-05 & $\times$ & 0.5 & $\times$ & 9.5 & 2.0 & 0.4 \\
SG05-10 & $\times$ & 1.0 & $\times$ & 9.0 & 2.0 & 0.4 \\
SG06B-01 & $\times$ & $\times$ & 0.1 & 9.9 & 2.0 & 0.4 \\
SG06B-02 & $\times$ & $\times$ & 0.2 & 9.8 & 2.0 & 0.4 \\
SG06B-03 & $\times$ & $\times$ & 0.3 & 9.7 & 2.0 & 0.4 \\
SG06B-04 & $\times$ & $\times$ & 0.4 & 9.6 & 2.0 & 0.4 \\
SG06B-05 & $\times$ & $\times$ & 0.5 & 9.5 & 2.0 & 0.4 \\
SG06B-10 & $\times$ & $\times$ & 1.0 & 9.0 & 2.0 & 0.4 \\
\hline
\end{tabular}

\subsubsection{Preparation Method}

A tin plate $(100 \times 50 \times 0.2 \mathrm{~mm})$ was polished with sandpaper and cleaned using alcohol. Using the wire rod applicator (Tianjin Zhongya Material Testing Machine Factory, Tianjin, China) to prepare the coating, the width of the wire rod was $80 \mu \mathrm{m}$. The specific operation was as follows: a glass rod was used to dip the resin onto the tin plate, and the wire rod was pulled from one end of the tin plate to the other end.

\subsection{Characterization}

Proton nuclear magnetic resonance $\left({ }^{1} \mathrm{H}-\mathrm{NMR}\right)$ was tested using an UAVANCEIII $400 \mathrm{MHZ}$, manufactured by Agilent (Santa Clara, CA, USA); $\mathrm{CDCl}_{3}$ was used as the solvent. ${ }^{1} \mathrm{H}-\mathrm{NMR}$ was also used to measure the $\mathrm{H}$ content of the hydrogen-containing polydimethylsiloxane by using the formula [17] below:

$$
E \%=\frac{B \times W_{s s} \times 8}{A \times W_{m s} \times 88} \times 100
$$

where $E \%$ is the content of the $\mathrm{H}, W_{s s}$ is the mass of the standard sample, $W_{m s}$ is the mass of the measurement sample, $B$ is the ${ }^{1} \mathrm{H}-\mathrm{NMR}$ integral area of the $\mathrm{Si}-\mathrm{H}$ proton peak of the hydrogen-containing polydimethylsiloxane, and $A$ is the ${ }^{1} \mathrm{H}-\mathrm{NMR}$ integral area of the dioxane. 
Fourier transform infrared (FT-IR) spectroscopy was obtained using a Bruker VERTEX70 FT-IR (Bruker, Ettlingen, Germany), with a $2 \mathrm{~cm}^{-1}$ resolution with 16 scanning cycles. Surface properties were determined using X-ray Photoelectron Spectroscopy (Axis Ultra DLD, Shimadza-Kratos, Manchester, UK) to measure the different resin surface elements, and the resin's surface contact angle was measured using a DSA20 video optical contact angle measuring instrument (Dataphysics, Filderstadt near Stuttgart, Germany). Then, Equations (2) and (3) were used to characterize the coating surface energy [15]:

$$
\begin{aligned}
& \gamma_{s l}=\frac{\gamma_{\mathrm{lg}}}{2}\left(\sqrt{1+\sin ^{2} \theta}+\cos \theta\right), 0 \leq \theta \leq \pi \\
& \gamma_{s g}=\frac{\gamma_{\mathrm{lg}}}{2}\left(\sqrt{1+\sin ^{2} \theta}+\cos \theta\right), 0 \leq \theta \leq \pi
\end{aligned}
$$

where $\gamma_{s l}$ is the surface energy of the solid and the liquid, $\gamma_{s g}$ is the surface energy of the solid and the gas, $\gamma_{\lg }$ is the surface energy of the liquid and the gas, and $\theta$ is the value of the contact angle.

\subsection{UV Curing Operation}

All samples were cured for $40 \mathrm{~s}$ and the samples were cured using a UV light source (RW-UVA-200U, Runwing, Taijin, China) at 250-400 nm with an irradiation dose of $40 \mathrm{~mW} / \mathrm{cm}^{2}$ on the surface of the samples.

\section{Results and Discussion}

\subsection{Synthesis and Characterization of Novel UV-Curable Fluorinated Siloxane Polymers}

These novel UV-curable fluorinated siloxane polymers were synthesized by stepwise reactions of AA, MEHQ, AIBN, AGE, mercaptoethanol, and hexafluorobutyl acrylate (HFOA), dodecafluoroheptyl acrylate (DFOA), and trifluorooctyl methacrylate (TFOMA), following the synthesis route shown in Figure 1.

\subsubsection{Characterization of Silicone Intermediates (H-PDMS, OH-PDMS, and EPDMS)}

The silicone intermediates of H-PDMS, OH-PDMS, and EPDMS were characterized by FT-IR and ${ }^{1} \mathrm{H}-\mathrm{NMR}$, as shown in Figures 2 and 3. We combined Equation (1) with the ${ }^{1} \mathrm{H}-\mathrm{NMR}$ of H-PDMS to calculate the $\mathrm{H}$ content of the hydrogen-containing polydimethysiloxane as $0.25 \%$.

The chemical structures of H-PDMS, EPDMS, and OH-PDMS were confirmed by FT-IR (Figure 2) and ${ }^{1} \mathrm{H}-\mathrm{NMR}$ (Figure 3). As shown in Figure 2, the wide and dispersive absorption peak around $3330 \mathrm{~cm}^{-1}$ demonstrated the presence of the -OH group in EPDMS. In addition, as shown in Figure 3 , the appearance of signals at the place of $\mathrm{f}$ and $\mathrm{g}$ and the peaks around $\delta 2.51-2.80 \mathrm{ppm}$, which are the methylene peaks belonging to the epoxy. A specific peak appeared around $\delta 2 \mathrm{ppm}$ in the spectrum of OH-PDMS and EPDMS, which can be ascribed to the -OH peak. These peaks showed that the epoxy group at the side-chain of the silicon oil intermediates had opened. The ${ }^{1} \mathrm{H}-\mathrm{NMR}$ spectrum of OH-PDMS showed the appearance of signals at the place of $\mathrm{h}$ and $\mathrm{I}$, and the peaks around $\delta$ 6.18-5.80 ppm are the $\mathrm{C}=\mathrm{C}$ peak that belongs to acrylic acid. The results further support our conclusion that EPDMS and OH-PDMS were successfully synthesized. 


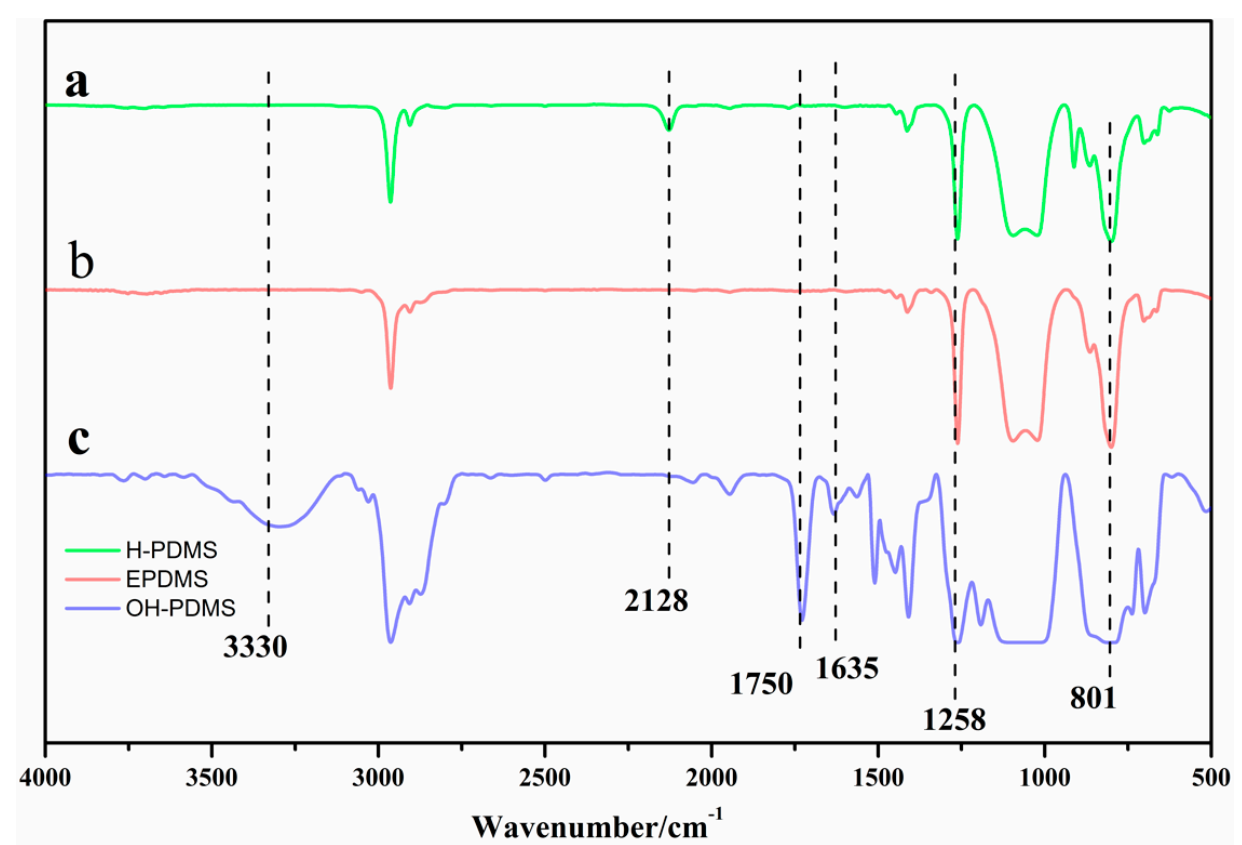

Figure 2. Fourier transform infrared (FT-IR) spectroscopy results of the intermediaries of fluorinated graft copolymer, where a is H-PDMS, b is EPDMS, and c is OH-PDMS.

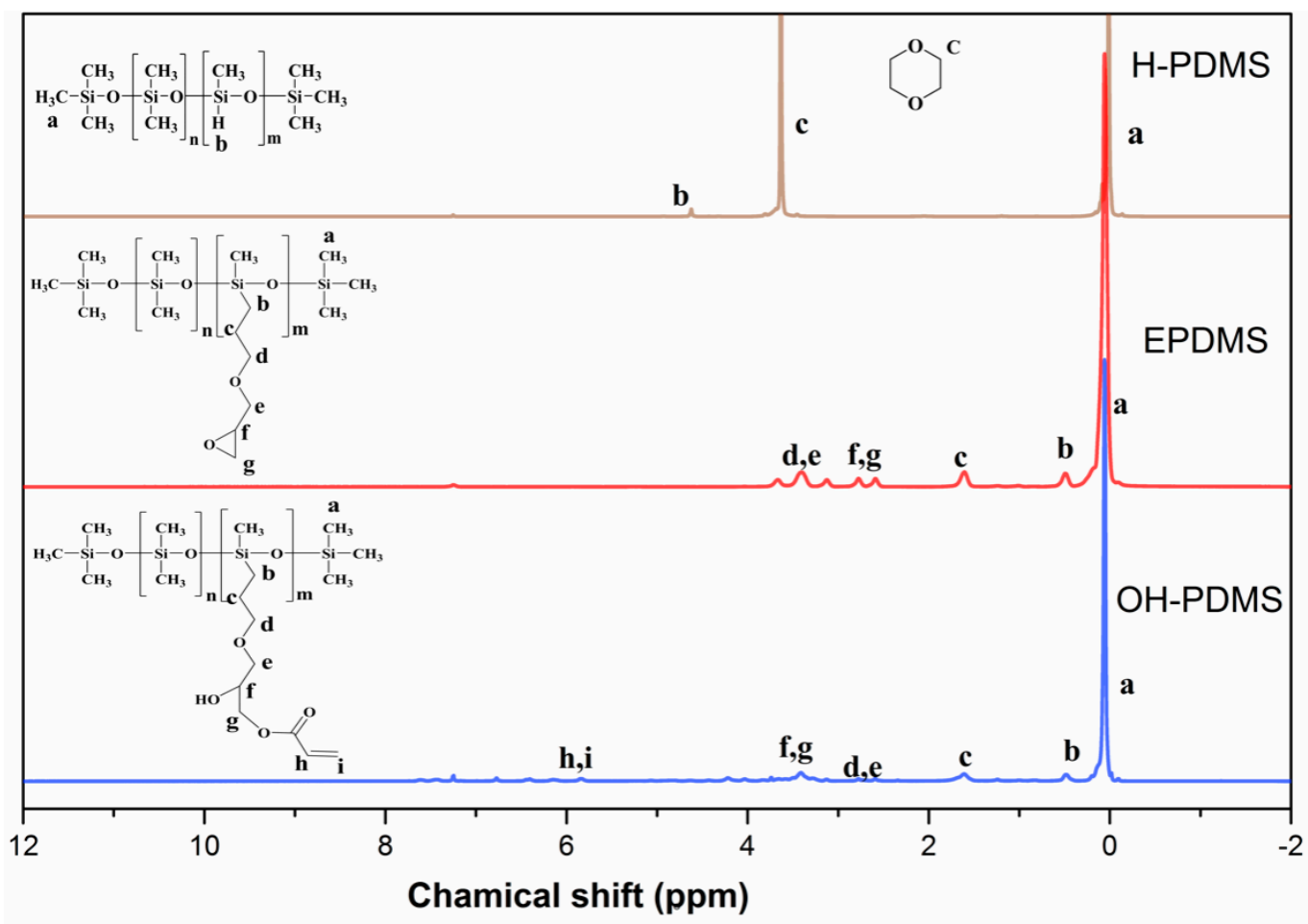

Figure 3. Proton nuclear magnetic resonance $\left({ }^{1} \mathrm{H}-\mathrm{NMR}\right)$ spectra of H-PDMS, OH-PDMS, and EPDMS.

\subsubsection{Characterization of Modified Fluorine-Containing Acrylic Acid}

As shown in Figure 4, the peaks at 1185, 1756, and $1280 \mathrm{~cm}^{-1}$ belong to the stretching vibration band of the $\mathrm{C}-\mathrm{O}-\mathrm{C}, \mathrm{C}=\mathrm{O}$, and $\mathrm{C}-\mathrm{F}$ groups, respectively. The wide and dispersive absorption peak at $3024 \mathrm{~cm}^{-1}$ belongs to the $-\mathrm{OH}$ group, and the peak of the $\mathrm{C}=\mathrm{C}$ group belongs to the acrylic acid appearing at 1644 and $807 \mathrm{~cm}^{-1}$. To further confirm the product, as shown in Figure 5, signals at $\delta$ 
1.16 ppm and $\delta 2.6-2.8$ ppm belong to the $-\mathrm{OH}$ group and $\mathrm{CH}_{2}-\mathrm{S}-\mathrm{CH}_{2}-\mathrm{CH}_{2}$, respectively. The results indicated that the product was successfully modified by acrylation.

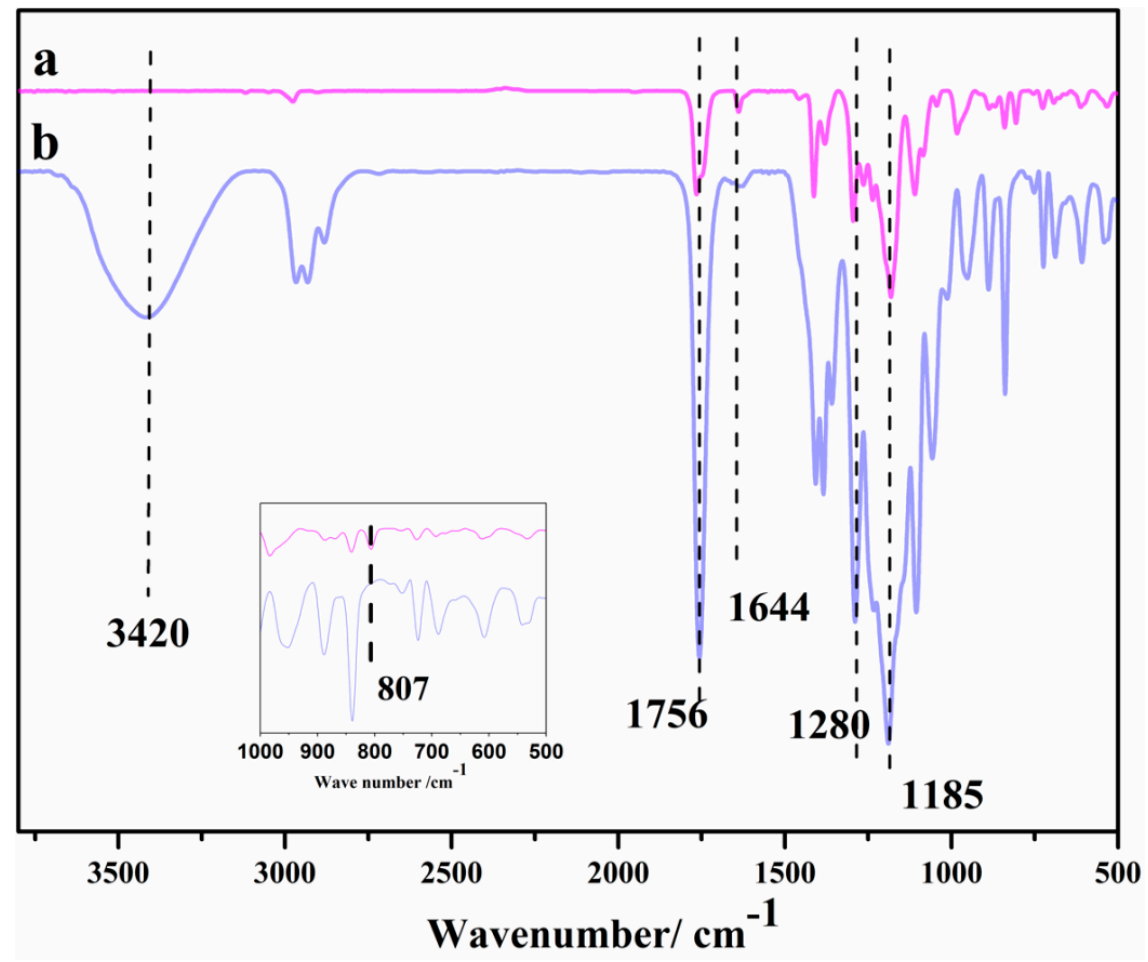

Figure 4. FT-IR of modified fluorine-containing acrylic acid: $a$, the HFOA; $b$, the copolymer reacted with mercaptan (OH-G01).

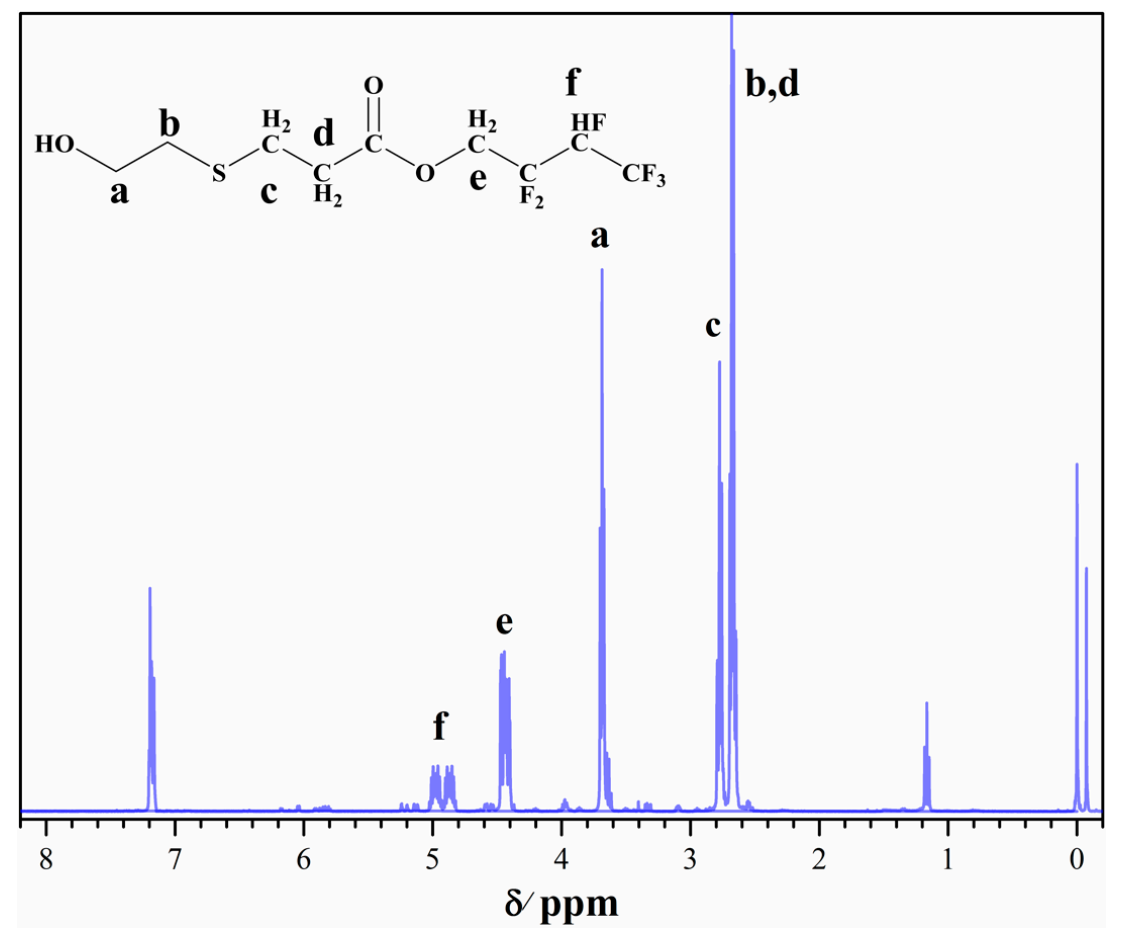

Figure 5. ${ }^{1} \mathrm{H}-\mathrm{NMR}$ spectra of $\mathrm{OH}-\mathrm{G} 01$. 


\subsection{UV-Curing Behavior}

The composition of SG01 resin is shown in Table 1. The UV-curing behavior was investigated by analyzing series of FT-IR spectroscopies. As shown in Figure 6, the descent of the peak at $1630 \mathrm{~cm}^{-1}$ of $\mathrm{C}=\mathrm{C}$, belonging to the acrylic acid stretching vibration bond, was observed after $10 \mathrm{~s}$ UV light irradiation. The peak at $1630 \mathrm{~cm}^{-1}$ is the indicator for the resin curing process. As the exposure time increased, the peak at $1630 \mathrm{~cm}^{-1}$ decreased slowly, until after $40 \mathrm{~s}$, where no further changes occurred. These FT-IR monitoring results clearly show that the coating contained a cross-linked network formed through acrylate polymerization, which prevented the movement of monomers. The results demonstrated that the fluoro-siloxane resin has excellent UV-cured performance.

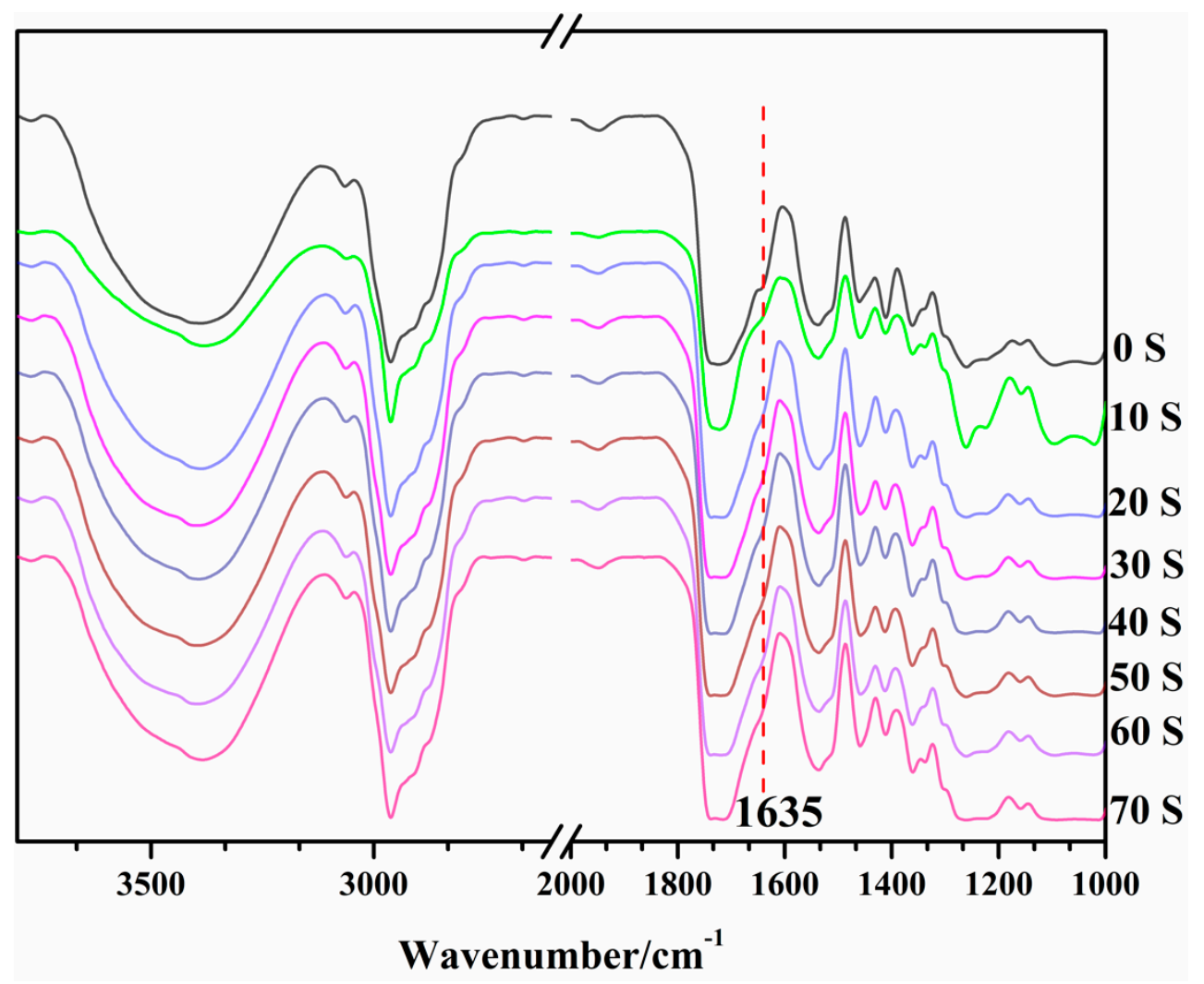

Figure 6. The UV-curable behavior of SG01 as monitored by FT-IR.

\subsection{Surface Characterization}

\subsubsection{X-Ray Photoelectron Analysis}

The surface information of fluorinated siloxane UV-cured resin on the air-polymer side was measured by XPS analysis. The peaks at 686, 149, and $99 \mathrm{eV}$ were assigned to F1s, Si2s, and Si2p respectively, as shown in Figure 7 and Table 2. Theoretically, the F element resin content should be $0.148 \%$ in SG05 resin. Actually, according to the measurement results, the F element content was much higher than the theoretical value by about 19-fold. Given this result, the data further demonstrate that $\mathrm{F}$ has the ability to easily migrate to the surface. Due to the $-\mathrm{CF}_{3}$ and $-\mathrm{CF}_{2}$ functional groups have low surface energy (surface energy of $\sim 15 \mathrm{mN} \cdot \mathrm{m}^{-1}$ and $\sim 25 \mathrm{mN} \cdot \mathrm{m}^{-1}$ respectively), the fluorine groups tended to migrate to the surface of the coatings, and the flexible siloxane also had a good tendency to move, thus the measurement values were higher than the theoretical values. Furthermore, with the content of fluoro-silioxane resin increasing, the surface F content was also augmented up to the saturation value, when the addition reached $0.3 \mathrm{~g}$ the $\mathrm{F}$ content did not notably change owing to 
the F enrichment achieving surface saturation and the F content no longer being able to migrate to the surface.



Binding Energy $(\mathrm{eV})$

Figure 7. X-ray photoelectron spectroscopy (XPS) of the SG05 series resin at different low-surface energy masses: $0.1 \mathrm{~g}, 0.3 \mathrm{~g}$, and $0.5 \mathrm{~g}$, denoted by SG05-01, SG05-03, and SG05-05, respectively.

Table 2. Comparison of theoretical and measured amounts of SG05 series.

\begin{tabular}{ccc}
\hline & Theory (\%) & Measurement (\%) \\
\hline SG05-01 & 0.148 & 2.86 \\
SG05-03 & 0.444 & 2.21 \\
SG05-05 & 0.740 & 2.79 \\
\hline
\end{tabular}

\subsubsection{Contact Angle and Surface Energy of the Resin}

The contact angles were measured to assess the wettability of Si-G01, Si-G05, and Si-G06B modified with DR-U356 resin. As shown in Figure 8, the pure DR-U356 resin contact angle was $59.41^{\circ}$. With the addition of fluorinated siloxane polymers at only $1 \mathrm{wt} \%$, the contact angle increased dramatically up to more than $90^{\circ}$. The reason for this phenomenon is that perfluoroalkyl terminal groups have a good ability to migrate to the surface, whereas flexible siloxane has a tendency to move. Therefore, when the fluorosilicone content is low, there is a significant effect on the contact angle of the resin. Since the fluorine-containing groups and the siloxane have very low surface energy, their enrichment on the surface induced extremely low surface energy and the resin was transformed from the hydrophilic to the hydrophobic state, as illustrated in Figure 9.

As shown in Figure 8, the SG06B series had a higher contact angle compared to the other two series resins. Firstly, the SG06B polymer had more fluorine-containing chains, leading to a more powerful ability to migrate to the surface. Secondly, the SG06B fluorine end group system tended to be out of the system and enter the air interface in an upright shape. Longer fluorine-containing segments can have more fluorine end groups protruding, which indirectly increases the fluorine content per unit area, so SG06B had the highest contact angle. The series of SG01 and SG05 had fewer fluoride chains and some of them are easily enwrapped by the siloxane segment. For the structure of G01 and G05 monomers, G05 has more branched-type fluorine end groups and G01 is a linear fluorine end group; they are different from G06B's linear fluorine end groups. Most of the G05 fluorine-containing segments were wrapped up in the system. The alkane cannot reach the surface, so the contact angles 
of the two groups were not much different. As shown in Figure 9, after being subjected to ultraviolet light for about $40 \mathrm{~s}$, the DR-U356 resin system formed a relatively dense crosslinked network structure. Some of the fluorine migrated to the surface of the matrix resin, and the other part was enwrapped inside the matrix resin.

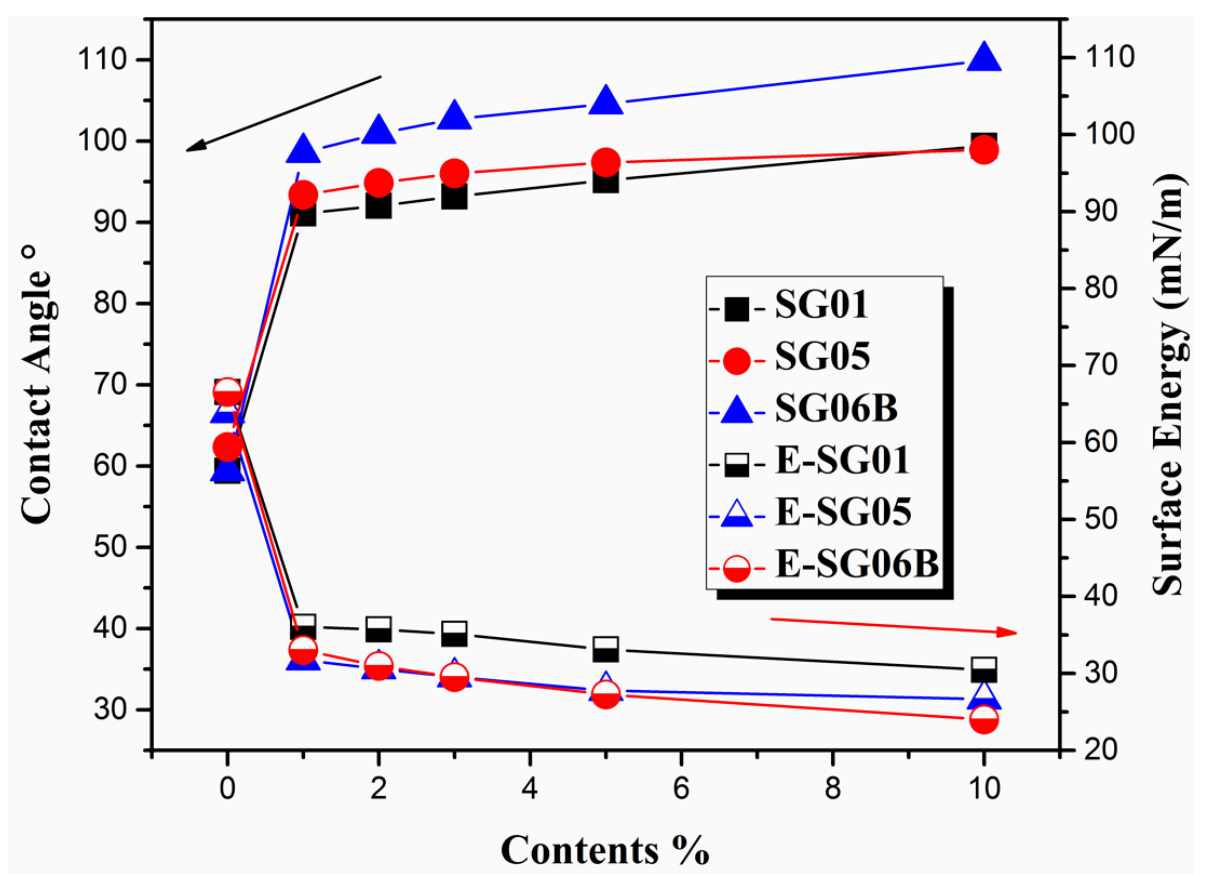

Figure 8. The contact angle and the surface energy of the resins.

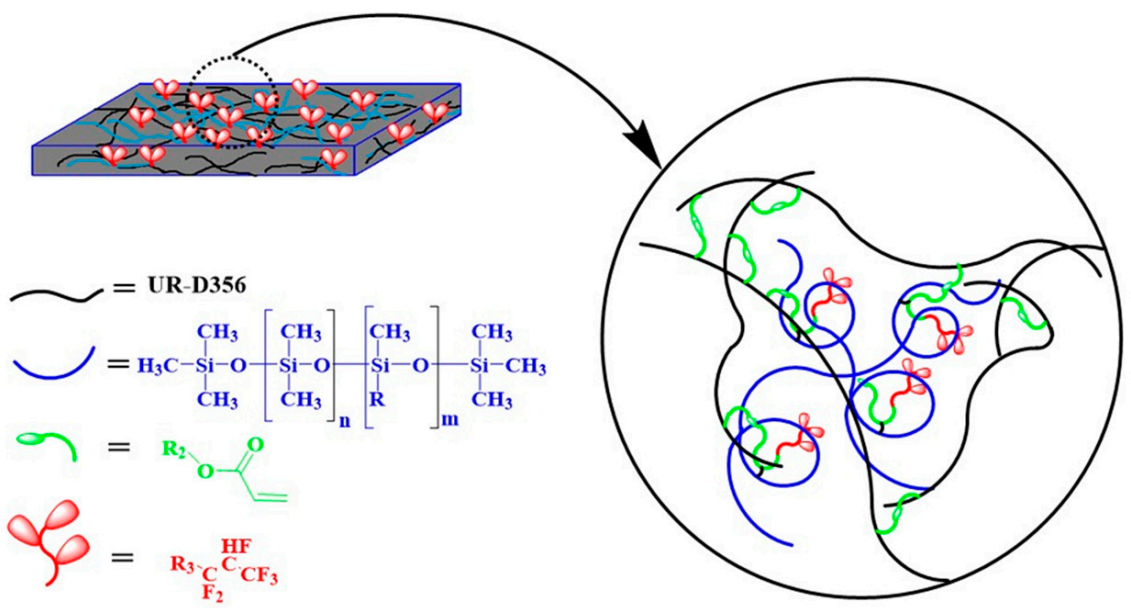

Figure 9. Schematic diagram of the micro-structure stage.

\subsubsection{Friction Recovering Properties}

Figure 10a-c demonstrate the friction recovering properties of the SG01-05, SG05-05, and SG06B-05 resins, respectively, examined using friction-annealing progressing. After being rubbed with 600 mesh sand paper about 10 times, the contact angle of the resins decreased below the original values. However, the SG01-05 and SG06B-05 resins, annealed under $120^{\circ} \mathrm{C}$ for $30 \mathrm{~min}$, demonstrated higher contact angles than the original values. SG05-05 only attained the original value. Compared to other films [18], SG01-05 and SG06B-05 resins showed excellent performance in terms of self-healing. 

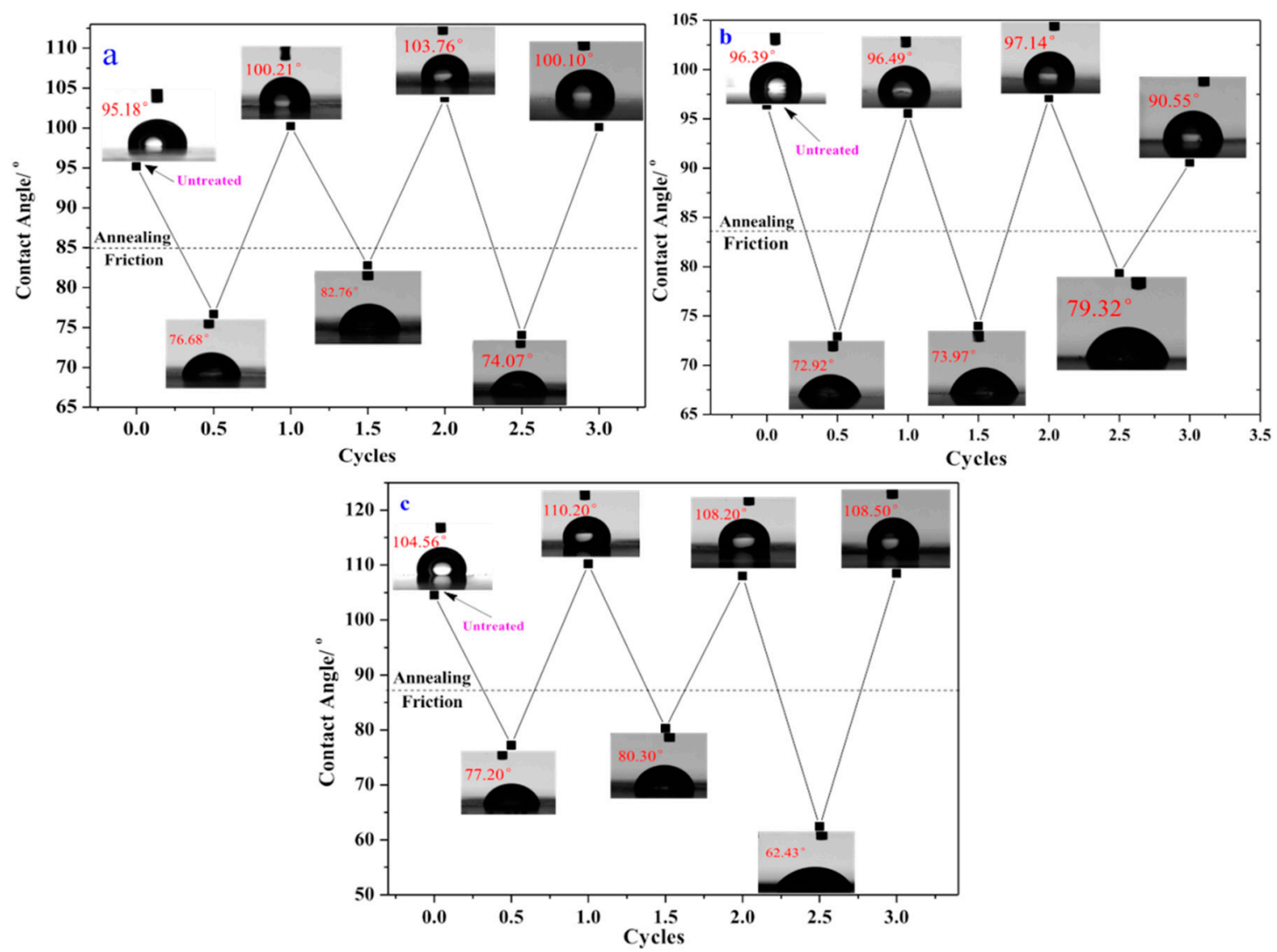

Figure 10. The friction recovering cycle test of the SG01-05 resin (a); The friction recovering cycle test of SG05-05 resin (b); The friction recovering cycle test of SG06B-05 resin (c).

The self-healing processing of the low surface energy resins (SG01-05 and SG06B-05) was described as follows: once the SG01-05 and SG06B-05 were rubbed with 600 mesh sandpaper, the F elements on the surface were either destroyed or removed. After SG01-05 and SG06B-05 annealed at $120{ }^{\circ} \mathrm{C}$ for $30 \mathrm{~min}$, the inner $\mathrm{F}$ elements migrated to the film surface to reduce the surface energy, driven by thermal energy. Even after three cycles of the friction-annealing process, SG01-05 and SG06B-05 maintained their excellent self-healing performance.

\subsection{Differential Scanning Calorimetry (DSC)}

The glass transition temperatures $\left(T_{g}\right)$ of the UV-cured resin were investigated by DSC. As shown in Figure 11, compared to the pure DR-U356 resin with the increase in the content of SG06B, the $T_{g}$ of the resins decreased. This suggests that the incorporation of fluorination destroyed the cross-linked density of pure DR-U356 resin. When the content of SG06B was more than $0.3 \mathrm{wt} \%$, the $T_{g}$ value decreased. The reason for this was that SG06B-03 had more and longer siloxane spacers than SG06B-01, so some fluorinated groups were buried by siloxane segments, which decreased the negative effect of destroying the crosslinking density of the pure DR-U356 resin. With the increasing SG06B polymer content, the resin system experienced phase segregation due to the poor compatibility between the SG06B polymer and pure DR-U356 resin. 


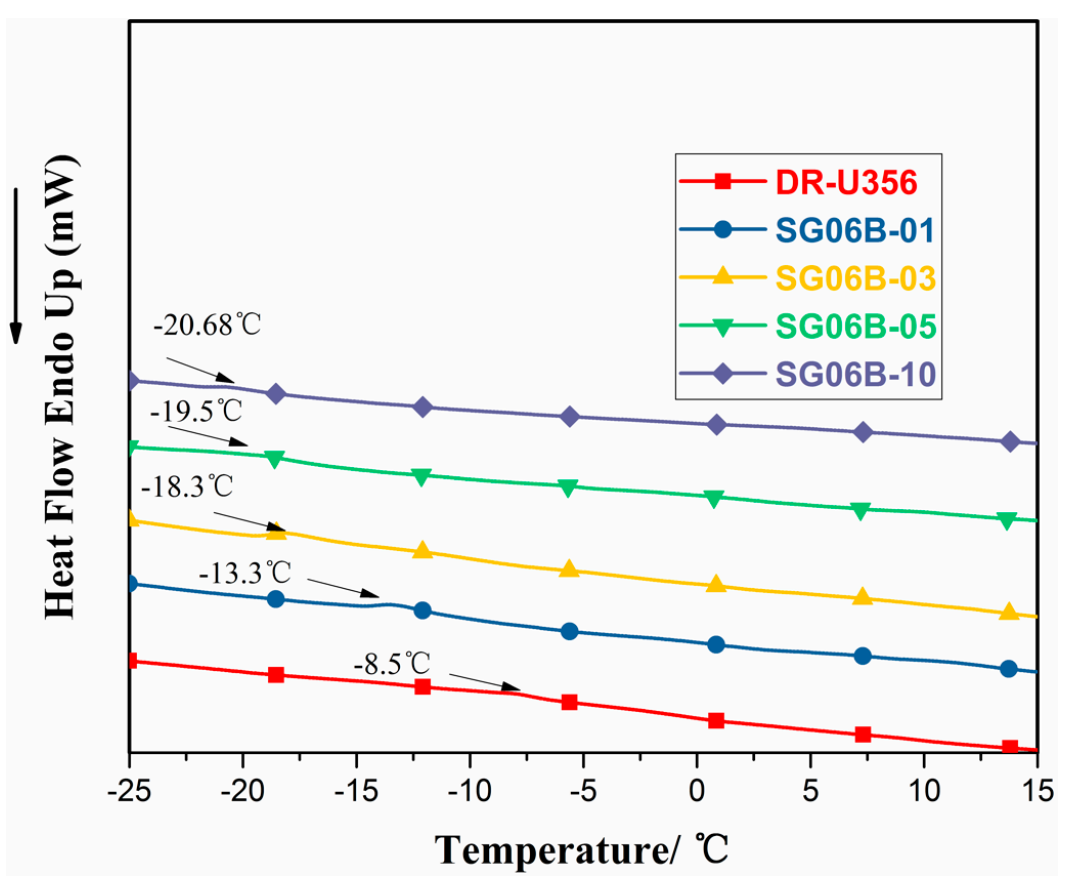

Figure 11. Differential scanning calorimetry (DSC) curves of different contents of SG06B graft UV-cured resin.

\subsection{Chemical Resistance}

As shown in Figure 12, three series resins added in the amount of one gram had excellent chemical resistance. The resistance to $10 \mathrm{wt} \% \mathrm{NaOH}$ solution corrosion was up to more than 10 times that of pure UR-D356 resin. The resistance to $10 \mathrm{wt} \% \mathrm{H}_{2} \mathrm{SO}_{4}$ solution was $49 \mathrm{~h}$, which is almost 20 times that of pure UR-D356 resin. This phenomenon can be explained as the siloxane and the fluoride chain segment have good inertness, which improves the stabilization of the resins. These results indicate that the addition of the fluoropolymer effectively improves the chemical resistance of the matrix resin.

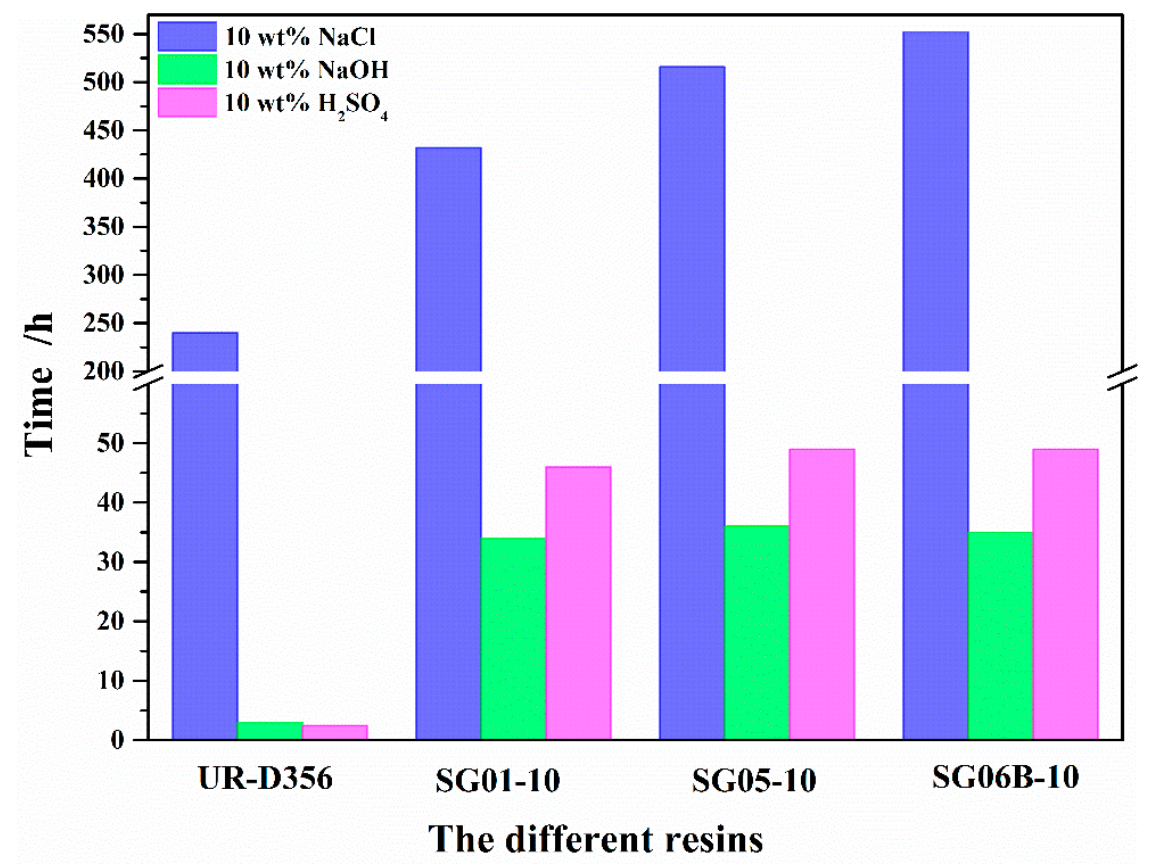

Figure 12. The bubble time of different resins after being dipped in salt, alkaline, and acid solution. 
As shown in Figure 13, when the addition was one or two grams, the resin's resistant destruction ability was weaker than the matrix resin. As part of this phenomenon, as the fluoro-siloxane resin has very low polarity and the polyurethane has very high polarity, they have poor miscibility. Therefore, the resin does not have a pyknotic surface. When the addition was increased to five grams, the chemical resistance notably improved with over $500 \mathrm{~h}$ of bubble time.

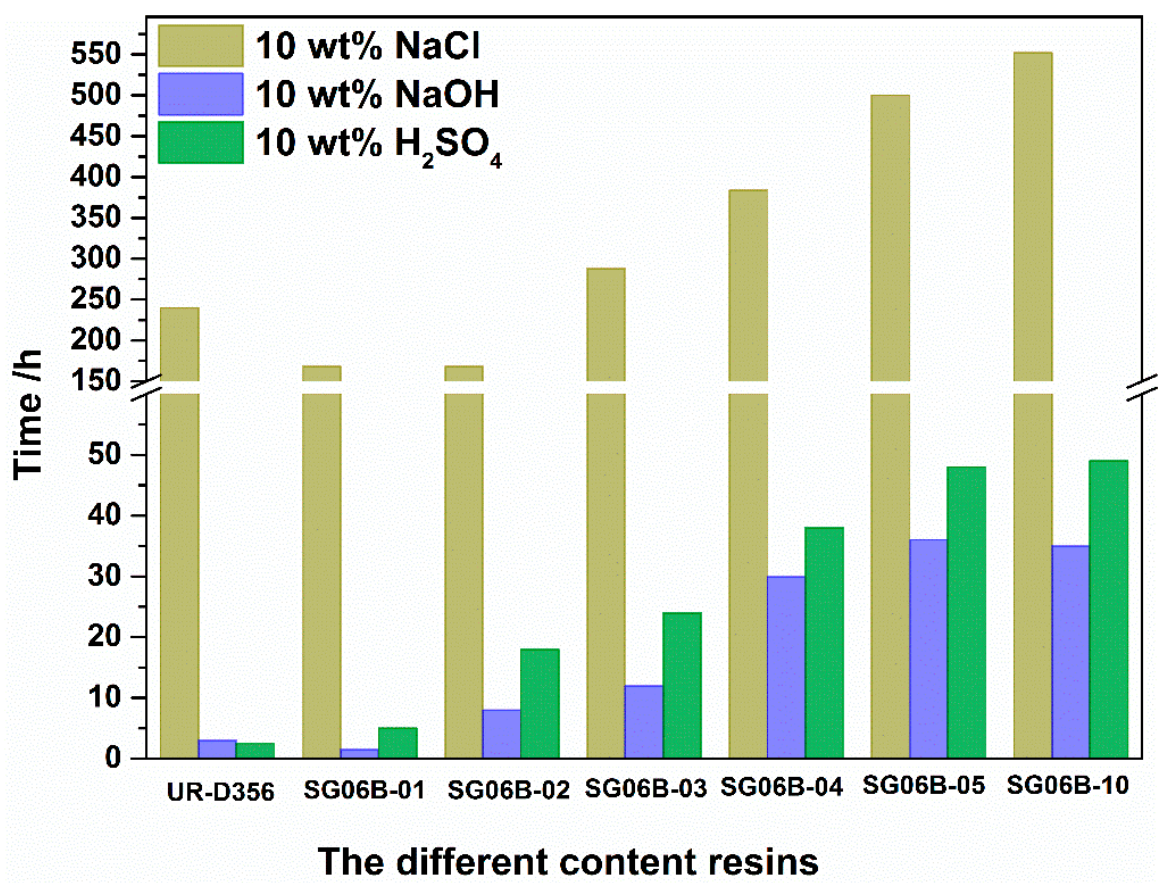

Figure 13. The bubble time with different contents of SG06B series polymers after being dipped in salt, alkaline, and acid solution.

\section{Conclusions}

In summary, three series of UV-curable fluorinated siloxane polymers (Si-G01, Si-G05, Si-G06B) were successfully synthesized. They were used as surface modifiers of UV-curable commercial polyurethane (DR-U356) at different concentrations (1, 2,3,4,5, and $10 \mathrm{wt} \%$ of DR-U356). Hydrophobic states $\left(91^{\circ}, 92^{\circ}\right.$, and $\left.98^{\circ}\right)$ were obtained at low concentrations $(1 \mathrm{wt} \%)$. The XPS results indicated that the fluorinated and siloxane groups were liable to migrate to the surface of resins, but with increasing fluoropolymer content, fluorine reached that saturation state on the surface. The incorporation of graft fluorinated siloxane polymers (Si-G01, Si-G05, and Si-G06B) in different amounts into commercial polyurethane (DR-U356) had a significant effect on the chemical resistance behavior of the cured polyurethane, since the fluorinated polymers had good chemical resistance. This work has many wide application prospects, such as water-resistant and anti-fingerprint applications, and provides a new strategy for building low surface energy materials or for corrosion resistant paint.

Author Contributions: Conceptualization C.Z., H.Y. and H.L.; Software, C.Z., Z.W., J.K., X.X., and J.D.; Validation, C.Z. and J.D.; Formal Analysis, C.Z. and J.D.; Investigation, J.D.; Resources, H.Y., H.L.; Data Curation, C.Z., L.X., J.Z. and J.D.; Writing-Original Draft Preparation, C.Z.; Writing-Review \& Editing, C.Z., H.Y., H.L. and W.X.; Visualization, C.Z., H.Y.; Supervision, H.L. and W.X; Project Administration, H.L. and W.X.; Funding Acquisition, H.L. and H.Y.

Funding: This research work was supported by the National Natural Science Foundation of China (No. 51103069, No. 51463017), Natural Science Foundation of Jiangxi Province (20171BAB206019), Science Foundation of Aeronautics of China (2016ZF56022), Scientific Research Foundation for the Returned Overseas Chinese Scholars ([2016]81), and the Department of Education fund of Jiangxi Province (No. GJJ170611) and the State Education Ministry and Scientific innovation fund of Nanchang Hang Kong University (YC2017001).

Conflicts of Interest: The authors declare no conflict of interest. 


\section{References}

1. Golovin, K.; Boban, M.; Mabry, J.M.; Tuteja, A. Designing self-healing superhydrophobic surfaces with exceptional mechanical durability. ACS Appl. Mater. Interface 2017, 9, 11212-11223. [CrossRef] [PubMed]

2. Sagisaka, M.; Narumi, T.; Niwase, M.; Narita, S.; Ohata, A.; James, C.; Yoshizawa, A.; Givenchy, E.T.D.; Guittard, F.; Alexander, S.; et al. Hyperbranched hydrocarbon surfactants give fluorocarbon-like low surface energies. Langmuir 2014, 30, 6057-6063. [CrossRef] [PubMed]

3. Alexander, S.; Smith, G.N.; James, C.; Rogers, S.E.; Guittard, F.; Sagisaka, M.; Eastoe, J. Low-surface energy surfactants with branched hydrocarbon architectures. Langmuir 2014, 30, 3413-3421. [CrossRef] [PubMed]

4. Wang, Y.; Shi, Y.; Pan, L.; Yang, M.; Peng, L.; Zong, S.; Shi, Y.; Yu, G. Multifunctional superhydrophobic surfaces templated from innately microstructured hydrogel matrix. Nano Lett. 2014, 14, 4803-4809. [CrossRef] [PubMed]

5. Buerkle, M.; Asai, Y. Thermal conductance of Teflon and Polyethylene: Insight from an atomistic, single-molecule level. Sci. Rep. 2017, 7, 41898. [CrossRef] [PubMed]

6. Major, L.; Lackner, J.M.; Kot, M.; Major, B. Bio-tribological properties and microstructure characterization of the polytetrafluorethylene (PTFE) coatings on polyaryletheretherketone (PEEK) substrate. Tribol. Int. 2016, 104, 309-320. [CrossRef]

7. Liao, H.; Zhang, B.; Huang, L.; Ma, D.; Jiao, Z.; Xie, Y.; Tan, S.; Cai, X. The utilization of carbon nitride to reinforce the mechanical and thermal properties of UV-curable waterborne polyurethane acrylate coatings. Prog. Org. Coat. 2015, 89, 35-41. [CrossRef]

8. Król, P. Synthesis methods, chemical structures and phase structures of linear polyurethanes. Properties and applications of linear polyurethanes in polyurethane elastomers, copolymers and ionomers. Prog. Mater. Sci. 2007, 52, 915-1015. [CrossRef]

9. $\mathrm{Li}, \mathrm{J} . ;$ Weng, R. Preparation of nano-SiO 2 /amino-modified polysiloxane hybrid superhydrophobic coating and thermal-stability characterization. J. Wuhan Univ. Technol. Mater. Sci. Ed. 2014, 29, 35-39. [CrossRef]

10. Yan, Z.; Liu, W.; Gao, N.; Wang, H.; Su, K. Synthesis and properties of a novel UV-cured fluorinated siloxane graft copolymer for improved surface, dielectric and tribological properties of epoxy acrylate coating. Appl. Surf. Sci. 2013, 284, 683-691. [CrossRef]

11. Yan, Z.; Liu, W.; Wang, H.; Su, K.; Hou, G. Synthesis and characterization of novel fluorinated siloxane star-like copolymer with short perfluoroalkyl chain and used for modification the epoxy resin. J. Fluor. Chem. 2014, 157, 63-72. [CrossRef]

12. Cappelletto, E.; Callone, E.; Campostrini, R.; Girardi, F.; Maggini, S.; Volpe, C.D. Hydrophobic siloxane paper coatings: The effect of increasing methyl substitution. J. Sol-Gel Sci. Technol. 2012, 62, 441-452. [CrossRef]

13. Tao, C.; Li, X.; Liu, B.; Zhang, K.; Zhao, Y.; Zhu, K.; Yuan, X. Highly icephobic properties on slippery surfaces formed from polysiloxane and fluorinated POSS. Prog. Org. Coat. 2017, 103, 48-59. [CrossRef]

14. Polizos, G.; Tuncer, E.; Qiu, X.; Aytuğ, T.; Kidder, M.K.; Messman, J.M.; Sauers, I. Nonfunctionalized polydimethyl siloxane superhydrophobic surfaces based on hydrophobic-hydrophilic interactions. Langmuir 2011, 27, 2953-2957. [CrossRef] [PubMed]

15. Yu, H.J.; Luo, Z.H. Novel superhydrophobic silica/poly (siloxane-fluoroacrylate) hybrid nanoparticles prepared via surface-initiated ATRP and their surface properties: The effects of polymerization conditions. J. Polym. Sci. Part A Polym. Chem. 2011, 49, 174-183. [CrossRef]

16. Momen, G.; Farzaneh, M. Facile approach in the development of icephobic hierarchically textured coatings as corrosion barrier. Appl. Surf. Sci. 2014, 299, 41-46. [CrossRef]

17. Oss, C.J.V.; Wu, W.; Docoslis, A.; Giese, R.F. The interfacial tensions with water and the Lewis acid-base surface tension parameters of polar organic liquids derived from their aqueous solubilities. Colloids Surf. B Biointerfaces 2001, 20, 87-91. [PubMed]

18. Wu, M.; An, N.; Li, Y.; Sun, J. Layer-by-layer assembly of fluorine-free polyelectrolyte-surfactant complexes for the fabrication of self-healing superhydrophobic films. Langmuir 2016, 32, 12361-12369. [CrossRef] [PubMed]

(C) 2018 by the authors. Licensee MDPI, Basel, Switzerland. This article is an open access article distributed under the terms and conditions of the Creative Commons Attribution (CC BY) license (http:/ / creativecommons.org/licenses/by/4.0/). 